\title{
Physical and Psychological Health Behavior Changes During the COVID-19 Pandemic that May Inform Surgical Prehabilitation: a Narrative Review
}

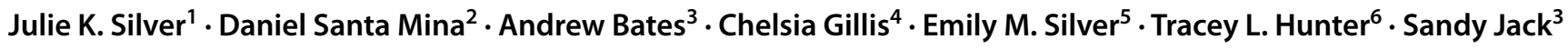

Accepted: 4 February 2022 / Published online: 18 February 2022

(c) The Author(s), under exclusive licence to Springer Science+Business Media, LLC, part of Springer Nature 2022

\begin{abstract}
Purpose of Review Multimodal prehabilitation aims to improve preoperative health in ways that reduce surgical complications and expedite post-operative recovery. However, the extent to which preoperative health has been affected by the COVID-19 pandemic is unclear and evidence for the mitigating effects of prehabilitation in this context has not been elucidated. The COVID-19 pandemic has forced a rapid reorganization of perioperative pathways. Delayed diagnosis and surgery have caused a backlog of cases awaiting surgery increasing the risk of more complex procedures due to disease progression. Poor fitness and preoperative deconditioning are predictive of surgical complications and may be compounded by pandemic-related restrictions to accessing supportive services. The COVID-19 pandemic has forced a rapid reorganization of perioperative pathways. This narrative review aims to summarize the understanding of the effects of the COVID-19 pandemic on preoperative health and related behaviors and their implication for the need and delivery for prehabilitation to engender improved surgical outcomes. A literature search of Medline was conducted for articles related to preoperative health, prehabilitation, and surgical outcomes published between December 1, 2020 and January 31, 2021. Additional hand searches for relevant publications within the included literature were also conducted through October 15, 2021. Recent Findings The COVID-19 pandemic, and measures designed to reduce the spread of the virus, have resulted in physical deconditioning, deleterious dietary changes, substance misuse, and heightened anxiety prior to surgery. Due to the adverse health changes prior to surgery, and often protracted waiting time for surgery, there is likely an elevated risk of peri- and post-operative complications. A small number of prehabilitation services and research programmes have been rapidly adapted or implemented to address these needs.

Summary During the COVID-19 pandemic to date, people undergoing surgery have faced a triple threat posed by extended wait times for surgery, reduced access to supportive services, and an elevated risk of poor outcomes. It is imperative that healthcare providers find ways to employ evidence-based prehabilitation strategies that are accessible and safe to mitigate the negative impact of the pandemic on surgical outcomes. Attention should be paid to cohorts most affected by established health inequities and further exacerbated by the pandemic.
\end{abstract}

Keywords Anesthesia $\cdot$ Anaesthesia $\cdot$ Rehabilitation $\cdot$ Prehabilitation $\cdot$ Physical and rehabilitation medicine $\cdot$ Perioperative care $\cdot$ Preoperative care $\cdot$ Preoperative period $\cdot$ Pandemic $\cdot$ COVID-19

\section{Introduction}

The COVID-19 global pandemic has had a profound and incalculable effect on surgical care and outcomes [1]. Originally identified in late 2019 in Wuhan, China, COVID-19

This article is part of the Topical Collection on Prehabilitation

Julie K. Silver

julie_silver@hms.harvard.edu

Extended author information available on the last page of the article quickly spread across the world, forcing health-resource reallocation and reprioritization, including surgical service reductions $[2,3,4]$. The ramp down of surgical procedures resulted in countless delayed diagnostic and curative surgeries [5•], including for people with a life-threatening diagnosis such as cancer [6]. While distress and deconditioning are common in the preoperative period, many patients awaiting surgery within the context of COVID-19 containment measures were at risk of additional obstacles to health maintenance, such as forced social distancing, lack of access to fitness facilities, disrupted and/or inadequate supply of 
nutritious food, financial hardship, worry about contracting the virus, and decreased access to healthcare. These and other issues may have occurred quickly and unexpectedly, intermittently, and often simultaneously to varying degrees, depending on timing and infectious disease rates, geographic location, public health action (or inaction), hospital and critical care resources, and vaccine availability.

Prior to the pandemic, it was known that people who are frail, elderly, and/or have poor functional capacity are at a higher risk for morbidity and mortality following elective surgery $[4,5 \bullet]$. Prehabilitation is recommended to reduce surgical risk in these populations and typically comprises a multimodal approach to reducing surgical vulnerability (e.g., physical fitness, nutritional status, mental health, etc.) $[1,7,8,9]$. Prehabilitation research is not new, and physically and psychologically preparing people for upcoming surgery is irrefutably beneficial; however, its value to patients and the health system may have markedly expanded during the COVID-19 pandemic given the negative impact of the pandemic on many health outcomes that can increase surgical risk. For example, prehabilitation may have a specific role in preventing deconditioning in those having to self-isolate during prolonged lockdowns or from infection with COVID-19. The goal of this report is to explore the recent literature pertaining to changes in preoperative health during the pandemic, how these changes adversely affect surgery, and how prehabilitation may be incorporated and adapted to improve patients' well-being and surgical outcomes.

\section{Methods}

A systematic search in PubMed of studies published between December 12020 and January 312021 was conducted using the following keyword search string: novel coronavirus OR COVID-19 OR SARS-CoV-2 OR Coronavirus AND prehabilitation OR diet OR nutrition OR stress OR anxiety OR psychological stress OR depression OR mental health OR vaping OR smoking OR smoking cessation OR alcohol OR increased alcohol OR alcohol use OR alcohol abuse OR substance use OR substance abuse OR opioid OR opioid use OR opioid abuse OR exercise OR rehabilitation OR physical activity. Articles reporting on the impact of the COVID-19 pandemic on preoperative health as it pertains to surgical risk or outcomes, as well as the role, delivery, and effect of prehabilitation contextualized to the pandemic were included and summarized by content experts as a narrative review. Additional hand searches for relevant publications within the included literature were also conducted through October 15, 2021.

\section{Preoperative Physical Activity, Exercise, and Fitness}

Much of the world has reported significant reductions in physical activity and increased sedentary behavior during the COVID-19 pandemic [10, 11••]. For example, Tison et al. assessed the step counts from over 450,000 people across 187 countries using app-based data from smartphones between January 2020 and June 2021 [11••]. They observed a nearly $30 \%$ reduction in step counts worldwide with variability in the timing and magnitude of reduction attributed to regional differences in infection rates and virus containment strategies. Reported pandemicrelated barriers to physical activity and exercise include COVID-19 containment measures such as social distancing, closure or use restrictions of healthcare and fitness facilities, concerns regarding contracting the SARS CoV2 virus while exercising in public, and costs associated with home-based exercise equipment $[12,13,14,15,16]$. The global reductions in physical activity and exercise are likely to contribute to reductions in physical fitness that is an independent predictor of surgical outcomes [17•]. Reduced physical fitness due to physical inactivity, combined with increased vulnerability to health ailments and chronic disease associated with sedentary behavior, will likely contribute to a "perfect storm" of increased perioperative risks for many patients undergoing elective surgery. This is exacerbated by the extended waiting times for surgery as procedure volume has been reduced to preserve resources for COVID-19 patients, creating a significant backlog of cases awaiting surgery [18].

Given the observed reductions in physical activity during the COVID-19 pandemic, research evaluating different strategies to recover physical activity and exercise levels is rapidly expanding [12]. Perhaps most notably, while rapid gains in its use were made prior to the pandemic, technology has emerged as an even more important component of assessment, instruction, engagement, and monitoring of people in exercise programs. Within the context of prehabilitation, virtual assessments have been used to develop individualized exercise prescriptions to optimize patient safety and treatment effect [6, 19, 20, 21]. For example, Sell and colleagues describe the use of a home-based 30-second sit-to-stand test/regimen monitored remotely by web-camera to assist with fitness level stratification and related exercise prescriptions [6]. In the UK, the SafeFit trial is investigating the use of virtual clinics to assess a person's exercise capacity, teach exercise routines, assess technique, and provide corrective feedback to enhance physical and emotional wellbeing for people with a cancer awaiting surgery [22]. In addition to connecting with exercise professionals virtually to support ongoing participation in exercise, the use of 
exercise gaming (exergaming) and personal apps may also play an important role in motivating and tracking physical activity volume and changes in fitness [23, 24, 25]. While the use of technology provides an important solution for many patients, it also raises important inequity considerations related to the accessibility of such care given the need for the devices, competence with technology, availability of reliable internet, and concerns regarding privacy for vulnerable populations.

The global reductions in physical activity and its impact on physical fitness will likely negatively affect surgery and recovery outcomes for many patients. Encouraging exercise for those awaiting surgery aligns with international guidance on physical activity during the pandemic and is strongly recommended. Given the potential increase in volume of patients awaiting surgery, screening for the most at-risk patients may be especially important, with self-report tools such as the Duke Activity Status Index (DASI) or the Godin-Shephard Leisure Time exercise questionnaire recommended to identify those with low functional capacity or physical activity, respectively [26, 26, 27, 28]. For at-risk patients and where in-person assessments are not possible, virtual connections between exercise professionals and patients may be used to evaluate patients for exercise safety and to provide individualized, home-based, or socially distant exercise prescriptions.

The role of prehabilitation for those patients with a history of contracting COVID-19 infection also requires special consideration given the potential impact of the disease that may include short- and long-term sequelae. These sequelae are multifactorial and may be termed "long COVID" or "post-acute sequelae of SARS CoV-2 (PASC)." Newly developed vaccines are also demonstrating important protective effects against severe disease and mortality. Effects of PASC may be especially though not exclusively, relevant to exercise capacity and tolerance. Research evaluating the relationship of physical activity, exercise, and fitness with COVID-19 outcomes is emerging, and early findings suggest physical fitness may provide a protective effect against COVID-19 severity and hospitalizations [29, 30, 31]. Exercise is known to have immune-modulating effects that may confer protection from COVID-19, and as such, studies of exercise interventions have been acknowledged as a research priority. Furthermore, there is emerging evidence of significant physical deconditioning after infection with COVID-19, which may respond to exercise interventions [32], though caution is warranted in post-viral conditions particularly if patients report more fatigue post-exercise [33••]. Exercise is also known to reduce risk factors that increase vulnerability to severe COVID-19-related morbidity, through its role in preventing and managing chronic disease [34,
35, 36]. Beyond the benefits of physical activity and exercise directly related to COVID-19 infection, the importance of these health behaviors on mental health and quality of life amidst pandemic containment measures, such as lockdowns and social distancing, is emerging rapidly [37, 38, 39]. Collectively, these established and novel areas of research have prompted urgent calls to action to promote and support safe physical activity and exercise during the pandemic, which may be especially relevant to those who are also awaiting surgery.

\section{Diet and Nutrition}

Improving diet and nutritional intake is a fundamental principle of prehabilitation. Nutritional prehabilitation serves to prevent or correct malnutrition, support functional exercise capacity, and optimize body composition [40, 41, 42]. The pandemic has created several nutrition challenges, including food insecurity and changes in dietary behavior, that ultimately affect nutritional health and body mass status. Disruption in the food supply chain (e.g., temporary closure of food processing facilities), increases in food prices, disruptions to social protection programs, altered food environments (e.g., restaurant closures during lockdown), instability in the employment and income of families, and social isolation (e.g., reduced communal meals) widen nutritional disparities and impact access to food, ultimately, contributing to a reduction in the quality and quantity of food consumed $[43,44]$. Furthermore, negative effects of confinement on dietary behavior and weight management have been well documented, globally. A multi-country electronic survey $(n=1047)$, comprised of several validated and crisis-oriented questionnaires, identified significant increases in sedentary behavior as well as self-perceived increases in consumption of unhealthy food, binge eating, snacking between meals, and increased total number of meals consumed per day during confinement [45]. A multi-center cross-sectional study of outpatients in Vietnam $(n=8291)$ identified that healthy eating scores were lower during lockdown, and that socioeconomic factors, including income and social status, were protective factors against the deterioration of these scores [46]. An Italian survey $(n=3533)$ revealed that nearly half of respondents observed weight gain during confinement [47]. A survey in Poland $(n=1097)$ found that increased snacking and weight gain had preferentially increased among the overweight and obese respondents, whereas those who were underweight reported further weight loss during confinement [48]. Similar findings were reported in an US survey $(n=7753)$, in which a greater portion of weight gain was reported in obese respondents during the pandemic. Interestingly, this survey found that scores for 
healthy eating increased due to a reduction in restaurant meals consumed [49]. A Spanish survey $(n=7514)$ also observed better eating habits among respondents with closer adherence to the Mediterranean diet during confinement [50]. Altogether, these findings suggest that the pandemic has largely brought negative changes in eating patterns and new nutrition challenges that could contribute to a worsening of nutritional status in those who are vulnerable as well as exacerbate losses in fat-free mass and gains in fat mass (i.e., enhanced sedentary behavior together with overconsumption of food).

Those who have been diagnosed with COVID-19 have additional nutrition challenges. Taste and olfactory impairments, including dysgeusia, were reported in $64 \%$ of a COVID-19 positive cohort; the majority experienced improvement in symptoms within 4 weeks of the initial diagnosis [51], but symptoms persisted in approximately $10 \%$ of the patients. Dysgeusia is a significant nutrition-impact symptom that impedes adequate food intake [52]. Additionally, rehabilitation post-COVID diagnosis represents another significant challenge [53]. The persistent fatigue characterized by many with COVID-19 can further promote sedentary behavior, which has several metabolic consequences [54]. Abrupt reduction in daily step count is associated with lean leg mass loss and promotion of insulin resistance [55]. Even in a young, healthy population, short-term immobilization was associated with losses of total thigh muscle volume by $1.7 \%$ and $5.5 \%$ of muscle volume after 2 and 7 days of disuse, respectively.

Given the reported effects of the pandemic, confinement, and a positive COVID-19 diagnosis on physical, nutritional, and mental health worldwide, the goal for nutrition prehabilitation during the pandemic may switch to simply maintaining nutritional status (rather than improving) to prevent further deterioration in metabolic status. For instance, attenuating the loss of fatfree mass and gain in fat mass that usually accompanies increased food intake and reduced physical activity may be a priority. Both sarcopenia and visceral obesity are associated with worse postoperative outcomes, including prolonged length of hospital stay and hospital readmissions [56]. Like prehabilitation exercises, remote nutritional consultations, assessment, and intervention, with shipment/delivery of nutritional supplementation if required, are likely to be essential components of nutritional prehabilitation within the pandemic. While it may not be possible to attain all nutrition measurements virtually, asking participants to periodically record their food intake and track their weight weekly is useful for monitoring progress and for identifying whether further nutrition support is required. The Patient-Generated Subjective Global Assessment can also be completed by a trained professional through a virtual platform (validation studies are in progress), and the continuous score offers an indication of improved/worsened nutritional status [57].

\section{Stress and Mental Health}

The need for psychological prehabilitation is growing as the impact of the COVID-19 pandemic impacts social, psychological, and emotional well-being worldwide. The implementation of measures that increase social isolation, such as regional or institutional lockdowns, has triggered increased psychological distress, including depressive and anxiety symptoms [58, 59]. A nationwide study in China revealed that over half of respondents rated the psychological impact of the pandemic as moderate to severe [60], and similar levels of psychological distress have been reported in Australia [61]. Increases in emotional eating related to stress [62] and the prevalence of sleep problems during the pandemic have also been observed [63], potentially indicative of disruptions in metabolic and circadian regulation due to psychological stress, which may affect healing. Distress and uncertainty related to pandemic concerns, such as worry for one's own or loved ones health, are likely to exacerbate both physical and psychological pathologies, impacting patient recovery [64].

As mental health undergoes new and exceptional challenges across all societies, the robust body of literature demonstrating the role of psychological factors on surgical outcomes has become more salient [65]. Indeed, evidence reflects pandemic-related stress and anxiety in patients waiting to undergo operations during the pandemic. Chronic pain patients report deterioration of selfreported pain, mental health, and ability to self-manage pain during indefinite postponement of elective spinal cord stimulation surgeries due to the pandemic [66]. Another study found that anxiety in neurosurgical patients awaiting non-urgent surgery is most strongly associated with concerns about contracting COVID-19, in addition to fears related to primary pathology, surgery, and disease worsening [67]. Bariatric surgery patients reported reduced quality of life and dietary compliance, along with high levels of pandemic-related worry [68]. Moreover, surgical patients may also be at a uniquely higher risk for psychological distress related to loneliness and isolation, as hospitals continue to implement visitor limitation policies [69]. Perceived loneliness and lack of social support are associated with higher rates of anxiety and depression, as well as risk of adverse physiological outcomes such as stroke or cardiac event [70].

Effective prehabilitation may therefore seek to integrate psychological interventions to buffer against negative effects of the pandemic and boost recovery by promoting 
positive psychological factors, including perceived social support. Favorable recovery may also be enhanced by selfefficacy and optimism [71], and growing evidence suggests perioperative psychological interventions effectively improve wound healing, innate and adaptive immunity, and mood [72]. Conversely, negative psychological factors (e.g., trait anxiety and depression) are associated with measures of impaired postoperative recovery, including slower wound healing and increases in cytokine-mediated inflammation, risk of postoperative complication, and length of hospital stay [71]. For example, cardiac patients with anxiety and depression are at higher risk for morbidity and mortality following coronary artery bypass graft surgery [60]. Psychological stress can exert direct control over neuroendocrine and inflammatory pathways to modulate immune responses, which may contribute to patterns of immune dysregulation following surgical stress and hence alter recovery trajectories $[1,5 \bullet]$. Ultimately, the interplay between positive and negative psychological factors contribute to the likelihood of favorable versus impaired surgical recovery (Fig. 1), where the pressures of the pandemic emphasize the importance of building psychological resilience.

\section{Tobacco, Alcohol, and Other Substance Use}

The COVID-19 pandemic has raised concern for increased risk of substance misuse as it may negatively impact physical function (e.g., respiratory status) and psychological health, and subsequently, impact efforts of surgical prehabilitation. In the USA, public health campaigning has decreased the prevalence of smoking; however, a significant percentage of the general population continue to use tobacco, which increases COVID-19 susceptibility and mortality [73, 74]. Smoking increases expression of angiotensin-converting enzyme-2, which appears to be linked to development of severe COVID-19 disease [73]. Some studies have found that increases in smoking during the pandemic are associated with younger age, lower education, second-hand exposure, or various psychosocial stressors $[75,76]$, whereas public awareness of the pandemic health crisis, including the negative outcomes of smoking on COVID-19 disease, incentivized many people to quit smoking [77, 78]. Additionally, government bans on tobacco sales in certain countries, such as South Africa and India, contributed to a reduction in tobacco
Fig. 1 Stress and surgery. This figure demonstrates how stress may impact surgery due to a host of physical and psychological factors and relationships

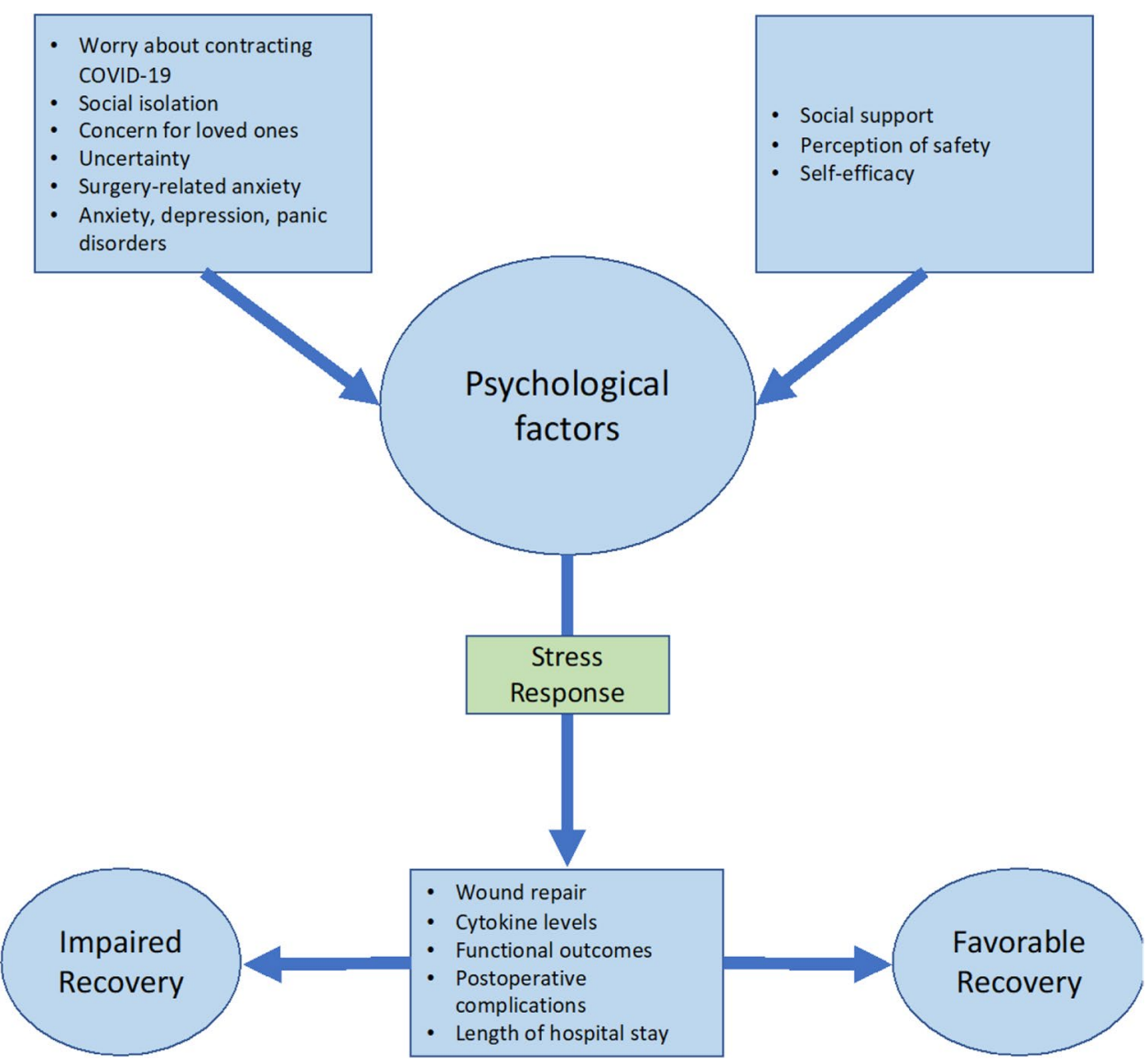


use [79]. Alcohol use during the pandemic has trended upwards in multiple countries, including Ireland, New Zealand, and France [80]. Proposed reasons included distress related to long-term social restrictions, potentially worsened symptoms of depression, anxiety, and a history of substance use disorder or mental health issues. As in prior global pandemics (e.g., the Severe Acute Respiratory Syndrome [SARS] outbreak in 2003), a decrease in alcohol consumption was expected during the COVID-19 pandemic likely related to financial limitations and reduced availability of alcohol purchase in select regions, such as Northern France and South Africa [79]. However, some high-income countries anticipated an increase in consumption as alcohol sales were maintained as essential business. In March 2020, national alcohol sales in the USA increased by $54 \%$ in comparison to the prior year, while online sales increased by $262 \%$ since 2019 [81]. The adverse effects of alcohol abuse include acute lung injury, neurotoxicity, and immune system dysfunction, which can reduce protection against the COVID-19 virus and increase risk of mortality [79, 82, 83].

Pandemic-related opioid use disorder has also been reported and has been associated with various socioeconomic stressors, as well as reduced healthcare access as priority was shifted to manage COVID-19 [84]. The Centers for Disease Control and Prevention reported over 81,000 opioid-induced deaths in the USA between May 2019 and 2020, corresponding to an $18 \%$ rise in overdose mortality [85]. This national drug epidemic influenced recent policy change by the US Department of Health and Human Services to reduce medical restriction on opioid use disorder management. Under new federal law, the exclusive "X-waiver" requirement for buprenorphine prescriptions was eliminated, expanding physician authority in opioid use disorder treatment to help decrease overdose trends [86]. However, buprenorphine is not an efficacious antidote for fentanyl, which is implicated in many overdose deaths [87]. To address this dilemma, Kosten et al. propose greater support and investment in antifentanyl vaccines, which potentially have superior relevance and efficacy to anticocaine and antinicotine vaccines that have been successfully used in the past 20 years [88].

Although studies are limited, opioid use disorder can result in respiratory depression and kidney injuries associated with poorer prognosis of COVID-19 infection [73]. The heightened risk of substance use disorder during the COVID-19 pandemic validates the need for improvement of public health education and medical access, in addition to destigmatization of addiction. Further research is required to understand the effects of substance use, particularly opioids and alcohol, on COVID-19 recovery and prehabilitation.

Pandemic-related changes in the use of tobacco, alcohol, and other substances may impact surgical outcomes (Table 1). Smoking cessation and substance abuse management in the pre-COVID-19 era has been an essential component of preoperative risk management $[1,89]$; and recognizing the association with respiratory disease is important.

\section{Discussion}

Perhaps now more than ever, emphasizing and supporting health behaviors as secondary prevention in addition to prehabilitation should be a public health priority. General declines in health and health behaviors have been observed globally, and for those who may require surgery, a worsening of pre-morbid or underlying health conditions have likely worsened during the pandemic for many people. For example, for some oncology patients, neo-adjuvant protocols were altered,

Table 1 Substance use during the COVID-19 pandemic

\begin{tabular}{llcc}
\hline & Tobacco Smoking & Alcohol & Opioids \\
\hline $\begin{array}{l}\text { Potential Correlates of } \\
\text { Increased Substance Use } \\
\begin{array}{l}\text { During the COVID-19 } \\
\text { Pandemic }\end{array}\end{array}$ & $\begin{array}{c}\text { Psychosocial stress, younger age, lower } \\
\text { education, second-hand exposure }\end{array}$ & $\begin{array}{c}\text { Psychosocial stress, mental health } \\
\text { issues, financial constraints, mainte- } \\
\text { nance of availability }\end{array}$ & $\begin{array}{c}\text { Psychosocial stress, limited } \\
\text { health care access, mental } \\
\text { health issues }\end{array}$ \\
$\begin{array}{l}\text { Potential Correlates of } \\
\text { Decreased Substance Use } \\
\begin{array}{l}\text { During the COVID-19 } \\
\text { Pandemic }\end{array}\end{array}$ & $\begin{array}{c}\text { COVID-19 health concerns, limited } \\
\text { availability }\end{array}$ & Limited availability & Limited availability \\
\hline
\end{tabular}

This table provides a summary of some of the issues related to substance use during the COVID-19 pandemic. Prehabilitation protocols should include evaluations of substance use and abuse. Smoking cessation is typically recommended prior to surgery. 
and operations delayed, putting them at higher risk for surgical complications and possible adverse oncologic outcomes [6]. Accordingly, routine screening and monitoring of patients over the preoperative period, however protracted it may be due to surgical backlogs, is likely very important to understand how the pandemic affected their health and surgical risk. These are also relevant to health systems as rapidly re-organized surgical services ramp up in the post-COVID period. Increases in surgical complications, extended length of stays, and increased needs for rehabilitation may assert significant strain upon already stressed healthcare systems. Accordingly, prehabilitation is gaining more attention as a standard of care component of surgical services and prehabilitation practice recommendations have recently been developed and endorsed by numerous perioperative organizations [90] (refer to Appendix Table 2 for an example). Numerous prehabilitation programs have adopted virtual models to meet the challenges of the pandemic and there is some evidence that these programs are feasible (high program adherence and patient satisfaction) [6, $19,21,22,91,92]$. Further to these recommendations and examples, we summarize additional resources for screening, assessment, intervention, and COVID-19-related considerations for prehabilitation (Table 2).

Beyond the benefits of a multimodal prehabilitation program that addresses surgical risk, exercise, nutrition, mental health, and the cessation of unhealthy behaviors (e.g., tobacco/substance abuse, sedentary behavior) may also have a direct impact on COVID-19 infection and outcomes. Moreover, prehabilitation has a role in the prevention and management of co-morbidities, including but not limited to, cardiopulmonary conditions, obesity, and diabetes, as these risk factors associate with higher morbidity and mortality in patients who develop COVID19 [93, 94]. Pandemic containment measures, such as lockdowns, infrastructure/facility restrictions, and social distancing also necessitate buffers against their negative impact on quality of life [37, 38, 39]. Thus, prehabilitation may have a role for the general population beyond surgery and include the prevention of severe COVID-19 outcomes [95].

It is important to consider that not all surgical patients will have comparable experiences, despite common health profiles and indications for surgery. Under usual circumstances, access to surgical care within a certain country or region may be affected by numerous factors such as patients' class, race, ethnicity, or gender [96, 97, 98]. Similarly, social determinants of health $(\mathrm{SDOH})$ such as health insurance, or lack thereof, may affect people's health and access to surgical care and post-surgical care such as rehabilitation [99, $100,101,102]$. During the pandemic, health disparities have been widely documented in the USA and numerous other countries [97, 98]. The COVID-19 pandemic has further exacerbated health inequities, with evidence of the requirement for hospitalization disproportionately affecting individuals from disadvantaged groups [103, 104], trends that have been observed previous infective outbreaks $[105,106]$. It is essential that healthcare professionals address these socio-economic and racial disparities or the negative the effects of the COVID-19 pandemic will amplify pre-existing health inequities. Members of disadvantaged groups are likely a priority group for whom prehabilitation should be offered given their likelihood of poorer health entering and exiting the operating room.

While this narrative review sheds light on the current COVID-19-pandemic challenges to health as they pertain to prehabilitation for surgery, this disease and its variants are rapidly evolving. Scientific advances in prevention (i.e., vaccines) and treatments are also rapidly evolving. Therefore, we can expect that there will be new and/or changing models of care that will have wide geographic reach and make prehabilitation easier to deliver to patients who will benefit from these interventions. The lessons we learn during the COVID-19 pandemic will support our understanding of flexible programming strategies and incorporate technology (e.g., virtual care) in the event of future pandemics. Notwithstanding the insights gained specific to pandemics, the lessons we learn will also apply to future perioperative care as we explore remote strategies to engage people who are not proximal to the usual infrastructure that support programs like prehabilitation [107, 108].

\section{Conclusion}

We report emerging evidence of global health and health behavior changes that may impact surgical outcomes. We further highlight adaptations to prehabilitation programs to support the changing and growing need for preoperative health optimization which may play an important role in managing protracted surgical waiting times and minimizing further burden on health systems. Ongoing assessment of innovations to prehabilitation delivery as well as attention to gaps in care that may disproportionately affect some groups is a high priority for research and quality assurance mandates. 


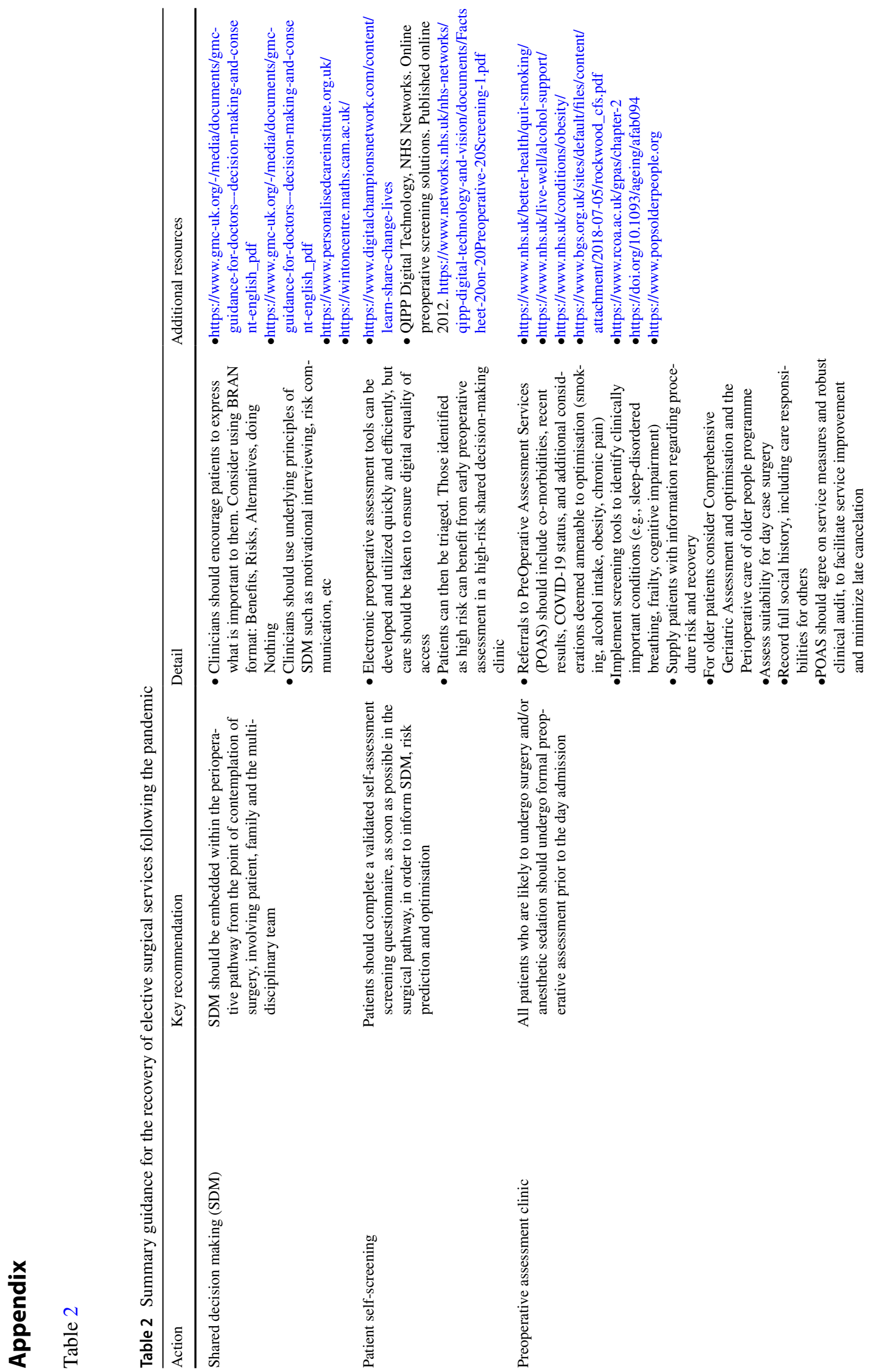




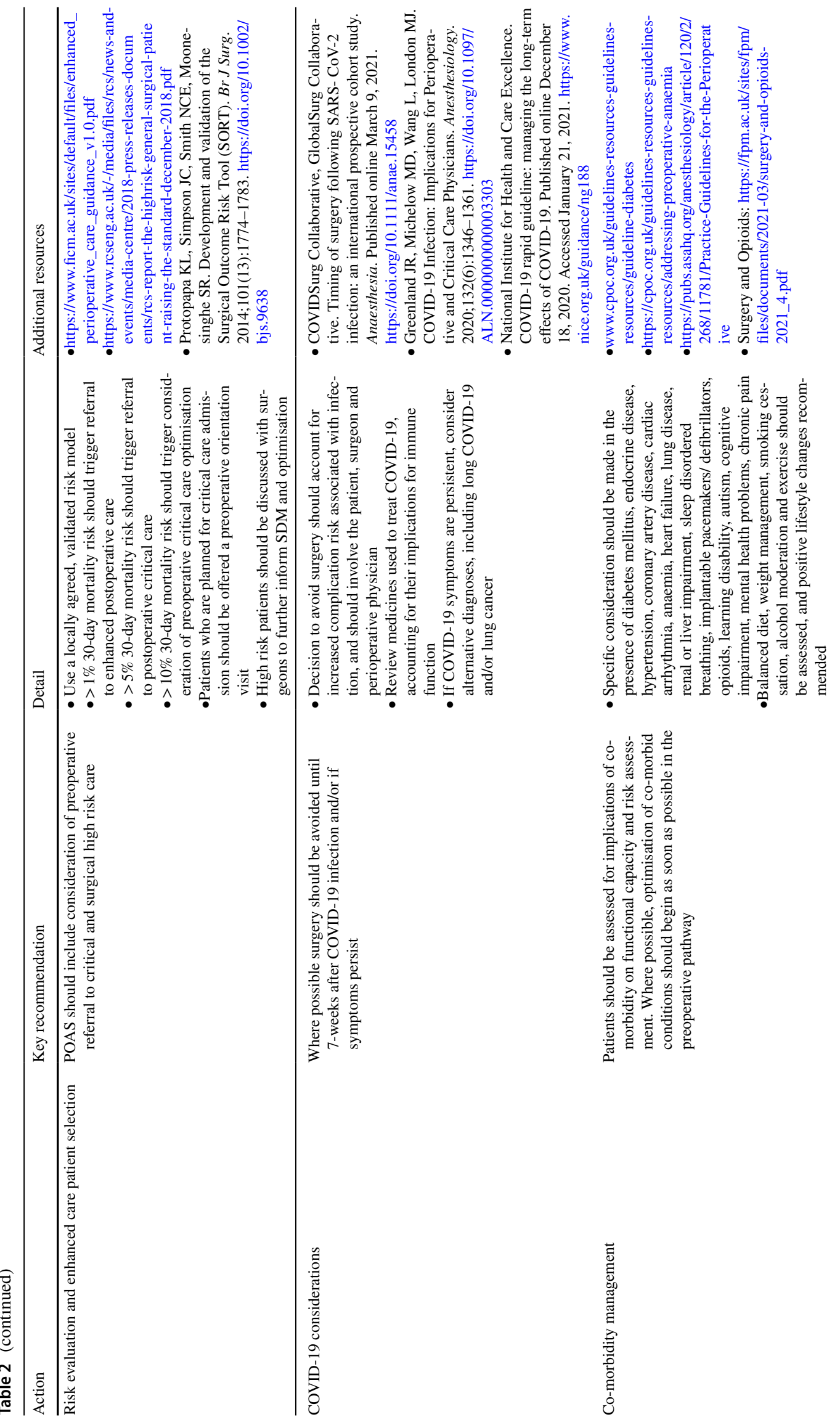




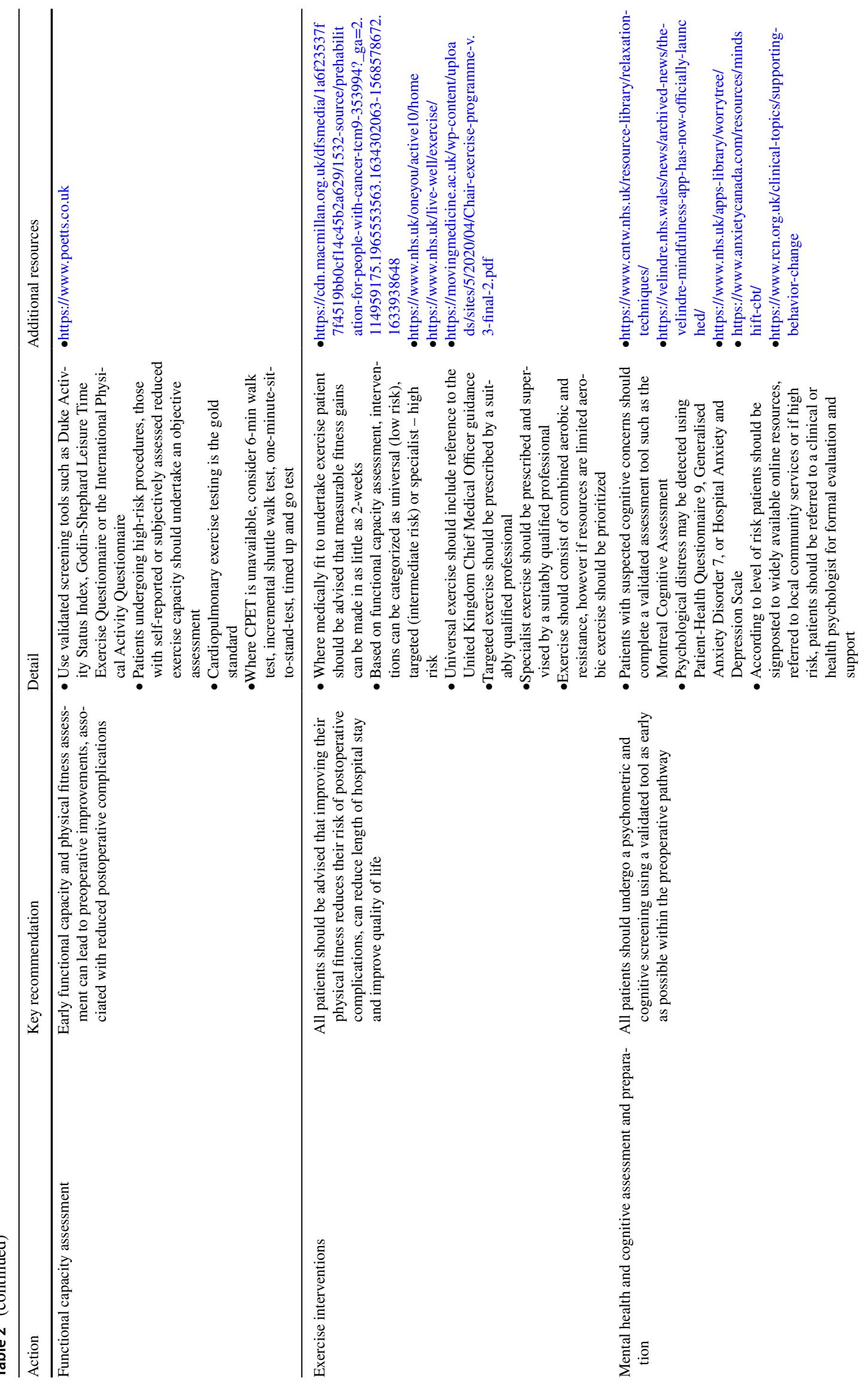




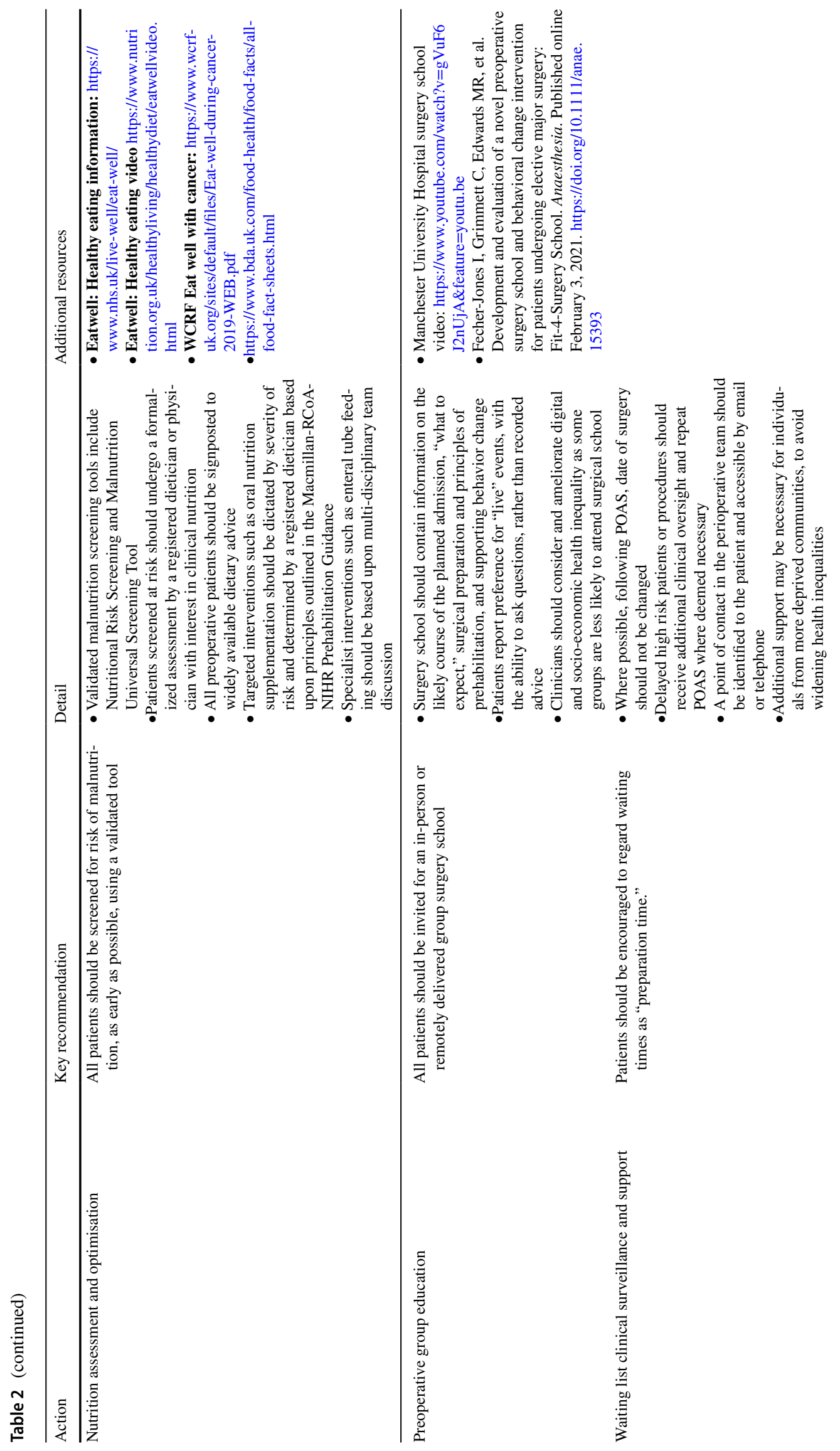


Acknowledgements The authors of this paper wish to recognize Prof. Denny Levett and Ms. Maggie Chen for their assistance with this paper.

Funding No funding was received for this work.

\section{Declarations}

Conflict of Interest JKS-no disclosures related to this work. Unrelated to this work, Dr. Silver has participated in research funded by the Arnold P. Gold Foundation and the Binational Scientific Foundation and is a venture partner at Third Culture Capital.

DSM-no disclosures related to this work.

SJ-no disclosures related to this work.

$\mathrm{AB}$ is funded by a National Institute for Health Research (NIHR) (predoctoral clinical academic fellowship). The views expressed are those of the author and not necessarily those of the National Health Service, the NIHR or the Department of Health and Social Care.

$\mathrm{CG}$-no disclosures related to this work.

EMS—no disclosures related to this work.

TLH-no disclosures related to this work.

Financial Benefits to Authors. The authors will not benefit financially from publication of this material.

Previous Presentation. This work has not been presented elsewhere in any form.

\section{References}

Papers of particular interest, published recently, have been highlighted as:

- Of importance

$\bullet$ Of major importance

1. Silver JK. Prehabilitation May Help Mitigate an Increase in COVID-19 Peripandemic surgical morbidity and mortality. Am J Phys Med Rehabil. 2020;99(6):459-63.

2. Sud A, Jones ME, Broggio J, Loveday C, Torr B, Garrett A, et al. Collateral damage: the impact on outcomes from cancer surgery of the COVID-19 pandemic. Ann Oncol. 2020;31(8):1065-74.

3. Ng JJ, Ho P, Dharmaraj RB, Wong JCL, Choong AMTL. The global impact of COVID-19 on vascular surgical services. J Vasc Surg. 2020;71(6):2182-3.e1.

4. Mazingi D, Navarro S, Bobel MC, Dube A, Mbanje C, Lavy C. Exploring the impact of COVID-19 on progress towards achieving global surgery goals. World J Surg. 2020;44(8):2451-7.

5. Richards M, Anderson M, Carter P, Ebert BL, Mossialos E. The impact of the COVID-19 pandemic on cancer care. Nature Cancer. 2020;1(6):565-7 (This perspective describes the impact of COVID-19 on cancer care during the initial part of the pandemic.).

6. Sell NM, Silver JK, Rando S, Draviam AC, Mina DS, Qadan M. Prehabilitation telemedicine in neoadjuvant surgical oncology patients during the novel COVID-19 coronavirus pandemic. Ann Surg. 2020;272(2):e81-3.

7. Bates A, West MA, Jack S. Framework for prehabilitation services. Br J Surg. 2020;107(2):e11-4.

8. Carli F, Silver JK, Feldman LS, McKee A, Gilman S, Gillis $\mathrm{C}$, et al. Surgical prehabilitation in patients with cancer: stateof-the-science and recommendations for future research from a 
panel of subject matter experts. Physical Medicine and Rehabilitation Clinics. 2017;28(1):49-64.

9. Sell NM, Qadan M, Silver JK. Implications of preoperative patient frailty on stratified postoperative mortality. JAMA Surg. 2020;155(7):669.

10. Caputo EL, Reichert FF. Studies of physical activity and COVID-19 during the pandemic: a scoping review. J Phys Act Health. 2020;17(12):1275-84.

11•• Tison GH, Avram R, Kuhar P, Abreau S, Marcus GM, Pletcher MJ, et al. Worldwide effect of COVID-19 on physical activity: a descriptive study. Annals of Internal Medicine. 2020;173(9):767-70 (This study included more than 450,000 unique users from 187 unique countries and assessed daily step count with the results demonstrating overall that steps counts decreased worldwide in the period after COVID-19 was declared a global pandemic. There were regional differences attributed to regional variation in COVID-19 timing, regional enforcement, and behavior change.)

12. Bentlage E, Ammar A, How D, Ahmed M, Trabelsi K, Chtourou $\mathrm{H}$, et al. Practical recommendations for maintaining active lifestyle during the COVID-19 Pandemic: a systematic literature review. Int J Environ Res Public Health. 2020;17(17):6265.

13. Ricci F, Izzicupo P, Moscucci F, Sciomer S, Maffei S, Di Baldassarre A, et al. Recommendations for physical inactivity and sedentary behavior during the coronavirus Disease (COVID-19) pandemic. Frontiers in Public Health. 2020;8(199).

14. Rhodes RE, Liu S, Lithopoulos A, Zhang CQ, Garcia-Barrera MA. Correlates of perceived physical activity transitions during the COVID-19 pandemic among Canadian adults. Appl Psychol Health Well Being. 2020;12(4):1157-82.

15. Denay KL, Breslow RG, Turner MN, Nieman DC, Roberts WO, Best TM. ACSM call to action statement: COVID-19 considerations for sports and physical activity. Current Sports Medicine Reports. 2020;19(8).

16. Rezende LFM, Lee DH, Ferrari G, Eluf-Neto J, Giovannucci EL. Physical activity for cancer patients during COVID-19 pandemic: a call to action. Cancer Causes \& Control. 2020.

17. Levett DZH, Jack S, Swart M, Carlisle J, Wilson J, Snowden C, et al. Perioperative cardiopulmonary exercise testing (CPET): consensus clinical guidelines on indications, organization, conduct, and physiological interpretation. British Journal of Anaesthesia. 2018;120(3):484-500 (This consensus report focuses on the use of perioperative cardiopulmonary exercise testing [CPET] to evaluate the risk of adverse perioperative events.).

18. Moletta L, Pierobon ES, Capovilla G, Costantini M, Salvador $\mathrm{R}$, Merigliano S, et al. International guidelines and recommendations for surgery during Covid-19 pandemic: a Systematic Review. Int J Surg. 2020;79:180-8.

19. Lambert G, Drummond K, Ferreira V, Carli F. Teleprehabilitation during COVID-19 pandemic: the essentials of "what" and “how." Support Care Cancer. 2021;29(2):551-4.

20. Verduzco-Gutierrez M, Bean AC, Tenforde AS, Tapia RN, Silver JK. How to conduct an outpatient telemedicine rehabilitation or prehabilitation visit. PM\&R. 2020;12(7):714-20.

21. Santa Mina D, Sellers D, Au D, Alibhai SMH, Clarke H, Cuthbertson $\mathrm{BH}$, et al. A pragmatic non-randomized trial of prehabilitation prior to cancer surgery: study protocol and COVID-19-related adaptations. Frontiers in Oncology. 2021;11(389):629207.

22. Grimmett C, Bates A, West M, Leggett S, Varkonyi-Sepp J, Campbell A, et al. SafeFit Trial: virtual clinics to deliver a multimodal intervention to improve psychological and physical wellbeing in people with cancer. Protocol of a COVID-19 targeted non-randomised phase III trial. BMJ Open. 2021;11(8):e048175.

23. Yang Y, Koenigstorfer J. Determinants of physical activity maintenance during the Covid-19 pandemic: a focus on fitness apps. Translational Behavioral Medicine. 2020;10(4):835-42.
24. Viana RB, de Lira CAB. Exergames as coping strategies for anxiety disorders during the COVID-19 quarantine period. Games Health J. 2020;9(3):147-9.

25. Ambrosino P, Fuschillo S, Papa A, Di Minno MND, Maniscalco M. Exergaming as a supportive tool for home-based rehabilitation in the COVID-19 pandemic era. Games Health J. 2020;9(5):311-3.

26. Wijeysundera DN, Beattie WS, Hillis GS, Abbott TEF, Shulman MA, Ackland GL, et al. Integration of the Duke Activity Status Index into preoperative risk evaluation: a multicentre prospective cohort study. Br J Anaesth. 2020;124(3):261-70.

27. Hlatky MA, Boineau RE, Higginbotham MB, Lee KL, Mark DB, Califf RM, et al. A brief self-administered questionnaire to determine functional capacity (the Duke Activity Status Index). Am J Cardiol. 1989;64(10):651-4.

28. Godin G, Shephard RJ. A simple method to assess exercise behavior in the community. Can J Appl Sport Sci. 1985;10(3):141-6.

29. Zhang X, Li X, Sun Z, He Y, Xu W, Campbell H, et al. Physical activity and COVID-19: an observational and Mendelian randomisation study. J Glob Health. 2020;10(2):020514.

30. Hamer M, Kivimäki M, Gale CR, Batty GD. Lifestyle risk factors, inflammatory mechanisms, and COVID-19 hospitalization: a community-based cohort study of 387,109 adults in UK. Brain Behav Immun. 2020;87:184-7.

31. Brawner CA, Ehrman JK, Bole S, Kerrigan DJ, Parikh SS, Lewis $\mathrm{BK}$, et al. Inverse relationship of maximal exercise capacity to hospitalization secondary to coronavirus disease 2019. Mayo Clin Proc. 2021;96(1):32-9.

32. Gao Y, Chen R, Geng Q, Mo X, Zhan C, Jian W, et al. Cardiopulmonary exercise testing might be helpful for interpretation of impaired pulmonary function in recovered COVID-19 patients. Eur Respir J. 2021;57(1):2004265.

33.• Herrera JE, Niehaus WN, Whiteson J, Azola A, Baratta JM, Fleming TK, et al. Multidisciplinary collaborative consensus guidance statement on the assessment and treatment of fatigue in postacute sequelae of SARS-CoV -2 infection ( PASC ) patients. PM\&R. 2021;13(9):1027-43 (This guidance statement on fatigue in PASC patients was developed by the American Academy of Physical Medicine and Rehabilitation's Multidisciplinary PASC Collaborative to provide recommendations and guidance from established PASC centers.).

34. Pedersen BK, Saltin B. Evidence for prescribing exercise as therapy in chronic disease. Scand J Med Sci Sports. 2006;16(S1):3-63.

35. Lavie CJ, Ozemek C, Carbone S, Katzmarzyk PT, Blair SN. Sedentary behavior, exercise, and cardiovascular health. Circ Res. 2019;124(5):799-815.

36. Anderson E, Durstine JL. Physical activity, exercise, and chronic diseases: a brief review. Sports Medicine and Health Science. 2019;1(1):3-10.

37. Faulkner J, O'Brien WJ, McGrane B, Wadsworth D, Batten $\mathrm{J}$, Askew CD, et al. Physical activity, mental health and wellbeing of adults during initial COVID-19 containment strategies: a multi-country cross-sectional analysis. J Sci Med Sport. 2021;24(4):320-6.

38. Feter N, Caputo EL, Smith EC, Doring IR, Cassuriaga J, Leite JS, et al. Association between physical activity and subjective memory decline triggered by the COVID-19 pandemic: Findings from the PAMPA cohort. Prev Med. 2021;145:106415.

39. Meyer J, McDowell C, Lansing J, Brower C, Smith L, Tully $\mathrm{M}$, et al. Changes in physical activity and sedentary behavior in response to COVID-19 and their associations with mental health in 3052 US adults. Int J Environ Res Public Health. 2020;17(18):6469. 
40. Gillis C, Wischmeyer PE. Pre-operative nutrition and the elective surgical patient: why, how and what? Anaesthesia. 2019;74:27-35.

41. Gillis C, Richer L, Fenton TR, Gramlich L, Keller H, CulosReed SN, et al. Colorectal cancer patients with malnutrition suffer poor physical and mental health before surgery. Surgery. 2021;170(3):841-7.

42. Gillis C, Hasil L, Kasvis P, Bibby N, Davies SJ, Prado CM, et al. Nutrition care process model approach to surgical prehabilitation in oncology. Frontiers in Nutrition. 2021;8(337).

43. Belanger MJ, Hill MA, Angelidi AM, Dalamaga M, Sowers JR, Mantzoros CS. Covid-19 and disparities in nutrition and obesity. New England Journal of Medicine. 2020;383(11):e69.

44. Nutrition CoWFSHLPoEoFSa. Impacts of COVID-19 on food security and nutrition: developing effective policy responses to address the hunger and malnutrition pandemic. Food and Agriculture Organization of the United Nations; 2020.

45. Ammar A, Brach M, Trabelsi K, Chtourou H, Boukhris O, Masmoudi L, et al. Effects of COVID-19 home confinement on eating behaviour and physical activity: results of the ECLB-COVID19 International Online Survey. Nutrients. 2020;12(6):1583.

46. Pham KM, Pham LV, Phan DT, Tran TV, Nguyen HC, Nguyen $\mathrm{MH}$, et al. Healthy dietary intake behavior potentially modifies the negative effect of COVID-19 lockdown on depression: a hospital and health center survey. Front Nutr. 2020;7:581043.

47. Di Renzo L, Gualtieri P, Pivari F, Soldati L, Attinà A, Cinelli G, et al. Eating habits and lifestyle changes during COVID-19 lockdown: an Italian survey. Journal of Translational Medicine. 2020;18(1).

48. Sidor A, Rzymski P. Dietary choices and habits during COVID-19 lockdown: experience from Poland. Nutrients. 2020;12(6): 1657.

49. Flanagan EW, Beyl RA, Fearnbach SN, Altazan AD, Martin CK, Redman LM. The impact of COVID-19 stayat-home orders on health behaviors in adults. Obesity. 2021;29(2):438-45.

50. Rodríguez-Pérez C, Molina-Montes E, Verardo V, Artacho R, García-Villanova B, Guerra-Hernández EJ, et al. Changes in dietary behaviours during the COVID-19 outbreak confinement in the Spanish COVIDiet study. Nutrients. 2020;12(6):1730.

51. Boscolo-Rizzo P, Borsetto D, Fabbris C, Spinato G, Frezza D, Menegaldo A, et al. Evolution of altered sense of smell or taste in patients with mildly symptomatic COVID-19. JAMA Otolaryngology-Head \& Neck Surgery. 2020;146(8):729.

52. Nolden AA, Hwang L-D, Boltong A, Reed DR. Chemosensory changes from cancer treatment and their effects on patients' food behavior: a scoping review. Nutrients. 2019;11(10):2285.

53. Kilroe SP, Fulford J, Jackman SR, Van Loon LJC, Wall BT. Temporal muscle-specific disuse atrophy during one week of leg immobilization. Med Sci Sports Exerc. 2020;52(4):944-54.

54. Humphreys H, Kilby L, Kudiersky N, Copeland R. Long COVID and the role of physical activity: a qualitative study. BMJ Open. 2021;11(3):e047632.

55. Oikawa SY, Holloway TM, Phillips SM. The impact of step reduction on muscle health in aging: protein and exercise as countermeasures. Front Nutr. 2019;6:75.

56. Martin L, Hopkins J, Malietzis G, Jenkins JT, Sawyer MB, Brisebois R, et al. Assessment of computed tomography (CT)-defined muscle and adipose tissue features in relation to short-term outcomes after elective surgery for colorectal cancer: a multicenter approach. Ann Surg Oncol. 2018;25(9):2669-80.

57. Bauer J, Capra S, Ferguson M. Use of the scored patientgenerated subjective global assessment (PG-SGA) as a nutrition assessment tool in patients with cancer. Eur J Clin Nutr. 2002;56(8):779-85.

58. Fiorillo A, Sampogna G, Giallonardo V, Del Vecchio V, Luciano $\mathrm{M}$, Albert U, et al. Effects of the lockdown on the mental health of the general population during the COVID-19 pandemic in Italy: Results from the COMET collaborative network. Eur Psychiatry. 2020;63(1):1-28.

59. Marazziti D, Pozza A, Di Giuseppe M, Conversano C. The psychosocial impact of COVID-19 pandemic in Italy: a lesson for mental health prevention in the first severely hit European country. Psychol Trauma. 2020;12(5):531-3.

60. Phillip JT, Robert AB. Depression, anxiety, and cardiac morbidity outcomes after coronary artery bypass surgery: a contemporary and practical review. J Geriatr Cardiol. 2012;9(2):197-208.

61. Rahman MA, Hoque N, Alif SM, Salehin M, Islam SMS, Banik $\mathrm{B}$, et al. Factors associated with psychological distress, fear and coping strategies during the COVID-19 pandemic in Australia. Globalization and Health. 2020;16(1).

62. Al-Musharaf S. Prevalence and predictors of emotional eating among healthy young Saudi women during the COVID-19 pandemic. Nutrients. 2020;12(10):2923.

63. Jahrami H, Bahammam AS, Bragazzi NL, Saif Z, Faris M, Vitiello MV. Sleep problems during the COVID-19 pandemic by population: a systematic review and meta-analysis. J Clin Sleep Med. 2021;17(2):299-313.

64. Tsamakis K, Triantafyllis A, Tsiptsios D, Spartalis E, Mueller C, Tsamakis C, et al. COVID-19 related stress exacerbates common physical and mental pathologies and affects treatment (Review). Experimental and Therapeutic Medicine. 2020.

65. Levett DZH, Grimmett C. Psychological factors, prehabilitation and surgical outcomes: evidence and future directions. Anaesthesia. 2019;74(Suppl 1):36-42.

66. Baranidharan G, Bretherton B, Eldabe S, Mehta V, Thomson S, Sharma ML, et al. The impact of the COVID-19 pandemic on patients awaiting spinal cord stimulation surgery in the United Kingdom: a multi-centre patient survey. Br J Pain. 2020;15(3):204946372094809.

67. Doglietto F, Vezzoli M, Biroli A, Saraceno G, Zanin L, Pertichetti M, et al. Anxiety in neurosurgical patients undergoing nonurgent surgery during the COVID-19 pandemic. Neurosurg Focus. 2020;49(6):E19.

68. Sisto A, Vicinanza F, Tuccinardi D, Watanabe M, Gallo IF, D'Alessio R, et al. The psychological impact of COVID-19 pandemic on patients included in a bariatric surgery program. Eating and weight disorders-studies on anorexia, bulimia and obesity. 2021;26(6):1737-1747.

69. Cazeau N. Social isolation: managing psychological distress in hospitalized patients during the COVID-19 pandemic. Clin J Oncol Nurs. 2020;24(5):472-4.

70. National Academies of Sciences E, Medicine. Social isolation and loneliness in older adults: opportunities for the health care system. Washington, DC: The National Academies Press; 2020. $316 \mathrm{p}$.

71. Mavros MN, Athanasiou S, Gkegkes ID, Polyzos KA, Peppas $\mathrm{G}$, Falagas ME. Do psychological variables affect early surgical recovery? PLoS ONE. 2011;6(5):e20306.

72. Villa G, Lanini I, Amass T, Bocciero V, Scirè Calabrisotto $\mathrm{C}$, Chelazzi C, et al. Effects of psychological interventions on anxiety and pain in patients undergoing major elective abdominal surgery: a systematic review. Perioperative Medicine. 2020;9(1).

73. Althobaiti YS, Alzahrani MA, Alsharif NA, Alrobaie NS, Alsaab HO, Uddin MN. The possible relationship between the abuse of tobacco, opioid, or alcohol with COVID-19. Healthcare. 2020;9(1):2

74. Salah HM, Sharma T, Mehta J. Smoking doubles the mortality risk in COVID-19: a meta-analysis of recent reports and potential mechanisms. Cureus. 2020.

75. Chen D. The psychosocial impact of the COVID-19 pandemic on changes in smoking behavior: Evidence from a 
nationwide survey in the UK. Tobacco Prevention \& Cessation. 2020;6(October):1-5.

76. Stanton R, To QG, Khalesi S, Williams SL, Alley SJ, Thwaite TL, et al. Depression, anxiety and stress during COVID-19: associations with changes in physical activity, sleep, tobacco and alcohol use in Australian adults. Int J Environ Res Public Health. 2020;17(11):4065.

77. Kayhan Tetik B, Gedik Tekinemre I, Taş S. The effect of the COVID-19 pandemic on smoking cessation success. J Community Health. 2021;46(3):471-5.

78. Streck JM, Kalkhoran S, Bearnot B, Gupta PS, Kalagher KM, Regan S, et al. Perceived risk, attitudes, and behavior of cigarette smokers and nicotine vapers receiving buprenorphine treatment for opioid use disorder during the COVID-19 pandemic. Drug Alcohol Depend. 2021;218:108438.

79. Rehm J, Kilian C, Ferreira-Borges C, Jernigan D, Monteiro M, Parry CDH, et al. Alcohol use in times of the COVID 19: implications for monitoring and policy. Drug Alcohol Rev. 2020;39(4):301-4.

80. Calvey T, Scheibein F, Saad NA, Shirasaka T, Dannatt L, Stowe MJ, et al. The changing landscape of alcohol use and alcohol use disorder during the COVID-19 pandemic-perspectives of early career professionals in 16 countries. J Addict Med. 2020;14(6):e284-6.

81. Wei Y, Shah R. Substance use disorder in the COVID-19 pandemic: a systematic review of vulnerabilities and complications. Pharmaceuticals. 2020;13(7):155.

82. Clay JM, Parker MO. Alcohol use and misuse during the COVID-19 pandemic: a potential public health crisis? The Lancet Public Health. 2020;5(5):e259.

83. Tran TD, Hammarberg K, Kirkman M, Nguyen HTM, Fisher J. Alcohol use and mental health status during the first months of COVID-19 pandemic in Australia. J Affect Disord. 2020;277:810-3.

84. Khatri UG, Perrone J. Opioid use disorder and COVID-19: crashing of the crises. J Addict Med. 2020;14(4):e6-7.

85. Dunlop A, Lokuge B, Masters D, Sequeira M, Saul P, Dunlop $\mathrm{G}$, et al. Challenges in maintaining treatment services for people who use drugs during the COVID-19 pandemic. Harm Reduction Journal. 2020;17(1).

86. Overdose deaths accelerating during COVID-19 [press release]. Centers for Disease Control and Prevention2020.

87. Practice Guidelines for the Administration of Buprenorphine for Treating Opioid Use Disorder. Federal Register; 2021.

88. Kosten TR, Petrakis IL. The hidden epidemic of opioid overdoses during the coronavirus disease 2019 pandemic. JAMA Psychiat. 2021;78(6):585.

89. Varga JT. Smoking and pulmonary complications: respiratory prehabilitation. J Thorac Dis. 2019;11(S5):S639-44.

90. Preoperative assessment and optimisation for adult surgery including consideration of COVID-19 and its implications. Centre for Perioperative Care; 2021.

91. Doiron-Cadrin P, Kairy D, Vendittoli PA, Lowry V, Poitras S, Desmeules F. Feasibility and preliminary effects of a teleprehabilitation program and an in-person prehablitation program compared to usual care for total hip or knee arthroplasty candidates: a pilot randomized controlled trial. Disabil Rehabil. 2020;42(7):989-98.

92. Piraux E, Caty G, Reychler G, Forget P, Deswysen Y. Feasibility and preliminary effectiveness of a tele-prehabilitation program in esophagogastric cancer patients. J Clin Med. 2020;9(7):2176.

93. Hoong CWS, Hussain I, Aravamudan VM, Phyu EE, Lin JHX, Koh H. Obesity is associated with poor Covid-19 outcomes: a systematic review and meta-analysis. Horm Metab Res. 2021;53(02):85-93.
94. Singh AK, Gillies CL, Singh R, Singh A, Chudasama Y, Coles $\mathrm{B}$, et al. Prevalence of co-morbidities and their association with mortality in patients with COVID-19: a systematic review and meta-analysis. Diabetes Obes Metab. 2020;22(10):1915-24.

95. Silver JK. Prehabilitation could save lives in a pandemic. BMJ. 2020:m1386

96. Best MJ, McFarland EG, Thakkar SC, Srikumaran U. Racial disparities in the use of surgical procedures in the US. JAMA Surg. 2021;156(3):274.

97. Connor J, Madhavan S, Mokashi M, Amanuel H, Johnson NR, Pace LE, et al. Health risks and outcomes that disproportionately affect women during the Covid-19 pandemic: a review. Social Science \& Medicine. 2020;266:113364.

98. Mackey K, Ayers CK, Kondo KK, Saha S, Advani SM, Young $\mathrm{S}$, et al. Racial and ethnic disparities in COVID-19-related infections, hospitalizations, and deaths: a systematic review. Ann Intern Med. 2021;174(3):362-73.

99. Zerhouni YA, Scott JW, Ta C, Hsu PC-H, Crandall M, Gale $\mathrm{SC}$, et al. Impact of the affordable care act on trauma and emergency general surgery: an Eastern Association for the Surgery of Trauma systematic review and meta-analysis. Journal of Trauma and Acute Care Surgery. 2019;87(2).

100. Baxter NB, Howard JC, Chung KC. A systematic review of health disparities research in plastic surgery. Plast Reconstr Surg. 2021;147(3):529-37.

101 Li HO-Y, Bailey AJ-M, Grose E, McDonald JT, Quimby A, Johnson-Obaseki S, et al. Socioeconomic status and melanoma in Canada: a systematic review. Journal of Cutaneous Medicine and Surgery. 2021;25(1):87-94.

102. Ziedas A, Abed V, Swantek A, Cross A, Chaides S, Rahman $\mathrm{T}$, et al. Social determinants of health influence access to care and outcomes in patients undergoing anterior cruciate ligament reconstruction: a systematic review. Arthroscopy. 2021.

103. Graboyes E, Cramer J, Balakrishnan K, Cognetti DM, LópezCevallos D, Almeida JR, et al. COVID -19 pandemic and health care disparities in head and neck cancer: Scanning the horizon. Head Neck. 2020;42(7):1555-9.

104. Siu JY. Health inequality experienced by the socially disadvantaged populations during the outbreak of COVID-19 in Hong Kong: an interaction with social inequality. Health Soc Care Community. 2021;29(5):1522-9.

105. Placzek H, Madoff L. Effect of race/ethnicity and socioeconomic status on pandemic H1N1-related outcomes in Massachusetts. Am J Public Health. 2014;104(1):e31-8.

106. Quinn SC, Kumar S, Freimuth VS, Musa D, Casteneda-Angarita N, Kidwell K. Racial disparities in exposure, susceptibility, and access to health care in the US H1N1 influenza pandemic. Am J Public Health. 2011;101(2):285-93.

107. Clemente-Suárez VJ, Dalamitros AA, Beltran-Velasco AI, Mielgo-Ayuso J, Tornero-Aguilera JF. Social and psychophysiological consequences of the COVID-19 pandemic: an extensive literature review. Frontiers in Psychology. 2020;11(3077).

108. Sepúlveda-Loyola W, Rodríguez-Sánchez I, Pérez-Rodríguez P, Ganz F, Torralba R, Oliveira DV, et al. Impact of social isolation due to COVID-19 on health in older people: mental and physical effects and recommendations. J Nutri Health Aging. 2020.

Publisher's Note Springer Nature remains neutral with regard to jurisdictional claims in published maps and institutional affiliations. 


\section{Authors and Affiliations}

\section{Julie K. Silver ${ }^{1} \cdot$ Daniel Santa Mina ${ }^{2} \cdot$ Andrew Bates $^{3} \cdot$ Chelsia Gillis $^{4} \cdot$ Emily M. Silver $^{5} \cdot$ Tracey L. Hunter $^{6} \cdot$ Sandy Jack $^{3}$}

Daniel Santa Mina

daniel.santamina@utoronto.ca

Andrew Bates

a.bates@soton.ac.uk

Chelsia Gillis

Chelsia.gillis@mcgill.ca

Emily M. Silver

esilver3@uchicago.edu

Tracey L. Hunter

Tracey.L.Hunter@kp.org

Sandy Jack

s.jack@soton.ac.uk

1 Harvard Department of Physical Medicine and Rehabilitation, Massachusetts General Hospital, 55 Fruit

Street, Boston, MA, USA
2 Faculty of Kinesiology and Physical Education, The University of Toronto, 55 Harbord St, Toronto, ON M5S 2W6, Canada

3 NIHR Southampton Biomedical Research Centre, Critical Care Research, University Hospital, Tremona Road, Southampton SO16 6YD, UK

4 School of Human Nutrition, McGill University, QC, Canada

5 Department of Psychology and Integrative Neuroscience Program, The University of Chicago, Chicago, IL, USA

6 Department of Internal Medicine, Kaiser Permanente Oakland Medical Center, Oakland, CA, USA 